\title{
$\mathbf{X}$ 射线安全检查技术研究新进展
}

陈志强 ${ }^{1,2^{*}}$, 张丽 $^{1,2}$, 金金金 1,2

1. 清华大学工程物理系, 北京 100084 ;

2. 粒子技术与辐射成像教育部重点实验室(清华大学), 北京 100084

*联系人, E-mail: czq@ mail.tsinghua.edu.cn

2016-06-14 收稿, 2016-07-06 修回, 2016-07-07 接受, 2017-02-23 网络版发表

国家杰出青年科学基金(11525521)、国家自然科学基金重点项目(11435007, 11235007)和国家重大科学仪器设备开发专项(2012YQ140037)资助

摘要暴恐事件的频发严重影响了国际社会的安全和秩序, 给客运、货运安全带来了前所未有的挑战. 有效打 击恐怖活动，保障人身、财产安全，是国内乃至全球公共安全领域的重要诉求。因此，如何通过高技术手段提升安 全检查能力，成为人们关注的重要问题和研究热点. 随着具有穿透能力的X射线技术的飞速发展，使用X射线对藏 匿于货物、行李及人体内外的爆炸物及违禁品进行检查，日益成为目前行之有效的技术手段。特别是近几十年来， 利用X射线所具有的多种与物质相互作用机制，研究和发展出多种查验技术，为公共安全提供了重要保障，表现 出极其广泛的应用前景. 本文对基于X射线的透视成像、散射成像、X-CT成像、能谱成像、衍射XRD、相祄成像 等安全检查技术做了全面介绍，针对主要技术的物理机制、应用特点及其研究进展进行了论述，并对新技术的研 究及应用作了展望, 以期进一步推动 $\mathrm{X}$ 射线技术在公共安全领域发挥更加重要的作用.

关键词安全检查, X 射线成像, CT 成像, 相祄成像, 衍射 XRD

美国9·11事件以来, 全球正不断遭受来自恐怖主 义的威胁, 针对民航、铁路、地铁车站等公共枢纽的 暴恐事件频发, 严重影响了国际社会的安全和秩 序 $^{[1,2]}$. 我国的反恐安全形势同样不容乐观, 2009年7 月乌鲁木齐发生的严重骚乱事件, 2012年6月新疆和 田恐怖劫机事件, 以及2014年3月的昆明火车站暴恐 事件等, 都对我国的反恐形势提出了严峻警示. 恐怖 主义的甚器尘上给客运、货运安全带来了前所未有的 挑战, 特别是在经济全球化发展的今天, 国际交流合 作对运输的需求正日益增长, 有效打击恐怖活动, 保 障人身、财产安全, 成为国内乃至全球高度关注的重 要问题. 安全形势的日益严峻, 使得对人体、行包、 货物等的安全性检查变得必要而迫切, 如何通过高 技术手段对藏匿于货物、行李及人体内外的爆炸物及 其构成材料进行有效检测, 是公共安全领域所面临
的新挑战.

1895年德国物理学家伦琴首次发现具有穿透能 力的 $\mathrm{X}$ 射线, 宣布了现代物理学时代的到来 ${ }^{[3]}$, 开启 了人类利用 $X$ 射线对物体内部结构进行成像的探 索. 早在1897年，巴黎火车站就使用X射线对手提行 李中的违禁品进行透视检查(图 1$)^{[4]}$, 这是 $X$ 射线技术 在安全检查领域的首次尝试.一百多年来，X射线技 术在放射诊疗、工业探伤、生物技术、公共安全等研 究领域发挥了举足轻重的作用，随着X射线源技术、 探测器技术、计算机与微电子技术和网络技术的飞速 发展, X射线透视成像、散射成像、X-CT成像、能谱 成像、衍射XRD、相衬成像等研究正不断深人, 极大 丰富和拓展了 $X$ 射线探测技术的内涵，成为安全检测 领域不可或缺的重要手段, 表现出极其广泛的应用 前景. 


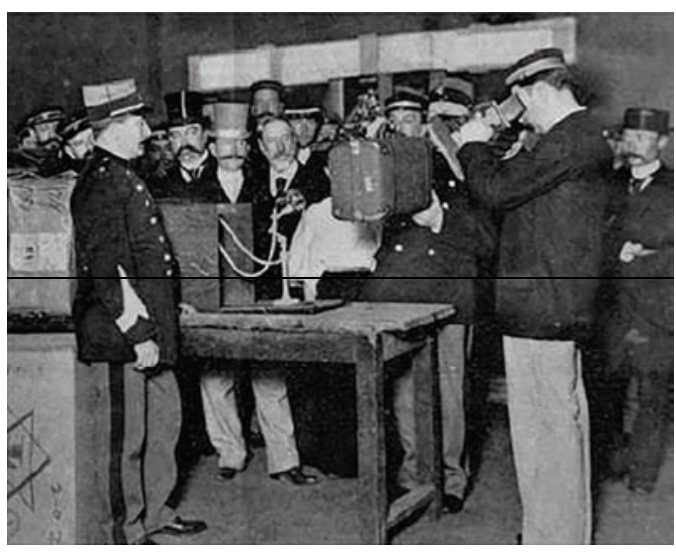

图 11897 年, 巴黎火车站使用X射线对旅客行包中的违禁品进行检 查 $^{[5]}$

Figure 1 Examination of a case in a Paris railway station in $1897^{[5]}$

\section{1 透视成像技术}

利用X射线和物质相互作用的物理机制, 可以有 效实现对违禁品的不开箱检查. X射线穿过物体时主 要发生瑞利散射、康普顿散射、光电效应和电子对效 应等相互作用, 造成射线强度的衰减(图2). 衰减程 度随着被穿透物质的成分和穿透路径长度的不同， 服从指数规律(Beer-Lambert定律):

$$
I=I_{0} \mathrm{e}^{-\int \mu_{m}(\bar{r}) \rho(\bar{r}) \mathrm{d} \bar{r}},
$$

其中 $I_{0}, I$ 分别为人射、出射射线强度, $\mu_{m}(\vec{r})$ 为位于坐 标 $\vec{r}$ 处的物质对人射 $\mathrm{X}$ 射线的质量衰减系数, $\rho(\vec{r})$ 为 物质密度. 对(1)式做对数变换, 可以得到最终的透 视成像公式:

$$
f(x, y)=\int_{L} \mu_{m}(\vec{r}) \rho(\vec{r}) \mathrm{d} \vec{r}=-\log \left(\frac{I}{I_{0}}\right),
$$

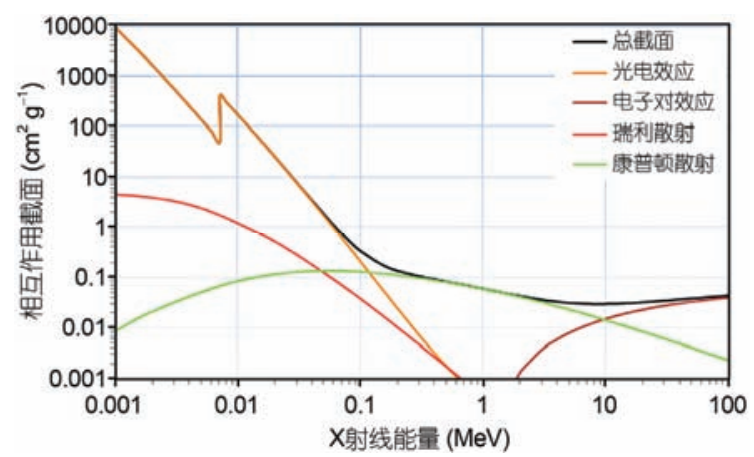

图 2 X射线和铁的 4 种相互作用截面曲线图

Figure $2 \mathrm{X}$-ray interaction cross section curves for iron
其中 $L$ 表示穿过图像上 $(x, y)$ 坐标点的射线路径，图 3 给出了使用 $X$ 射线进行透视成像的原理示意. 在透视 成像中, 图像的对比度主要由路径上物质的质量衰 减系数、物质密度以及X射线的透射距离这 3 个因素 决定. 这种特点使得透视成像对于高密度、强 $\mathrm{X}$ 射线 吸收能力的物质具有显著的辨识能力, 对实现行李 物品中的金属类违禁品, 特别是枪支、刀具、手雷、 雷管等物品的检查, 具有重要作用.

由于 $X$ 射线透视图像同时反映了物体成分及透 射厚度, 传统单能 X射线源的透视成像较难实现这些 物理量的有效区分. 采用双能X射线进行探测, 通过 物质对高、低能两种 $\mathrm{X}$ 射线的不同衰减情况，可以一 定程度上消除透射厚度的影响, 实现对物质成分的 粗略估计. 对给定的高、低能人射 X射线, 联合公式 (1) 和(2)可以得到高、低能透视图像 $f_{H i}(x, y), f_{L o}(x, y)$, 定义 $R$ 为二者之比, 则有

$$
R=\frac{f_{L o}(x, y)}{f_{H i}(x, y)}=\frac{\int_{L} \mu_{m, L o}(\vec{r}) \rho(\vec{r}) \mathrm{d} \vec{r}}{\int_{L} \mu_{m, H i}(\vec{r}) \rho(\vec{r}) \mathrm{d} \vec{r}}=\frac{\overline{\mu_{m, L o}}}{\overline{\mu_{m, H i}}},
$$

其中 $\overline{\mu_{m, L o}}$ 和 $\overline{\mu_{m, H i}}$ 为射线路径上的平均高、低能质量 衰减系数. 图 4 给出了在不同X射线能量下 $(60$ 和 100 $\mathrm{keV}) R$ 值随物质原子序数 $Z$ 的变化情况. 图中可以看 出, 在 $Z$ 小于 30 的范围内, $R-Z$ 曲线有着非常好的单调 特性, 因此当物体重叠情况不严重时, 双能透视系统 能够很好地实现对不同物质的分类, 根据原子序数 (混合物计算其等效原子序数)将其分为有机物、有机 /无机混合物、无机物及重金属等不同类别，获得丰富 的图像信息. 图 5 为单双能透视成像对比, 不难看出, 双能图像的分类着色使不同物质更容易区分，有效 降低了辨识难度, 对于提升查验准确率有着非常显 著的效果.

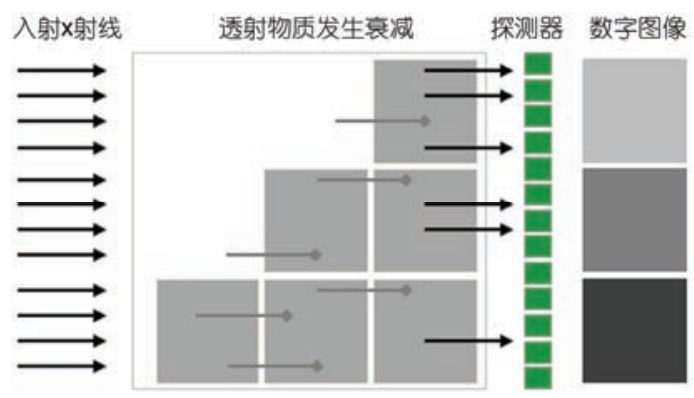

图 3 X射线透视成像原理示意图

Figure 3 Diagram of X-ray transmission imaging 


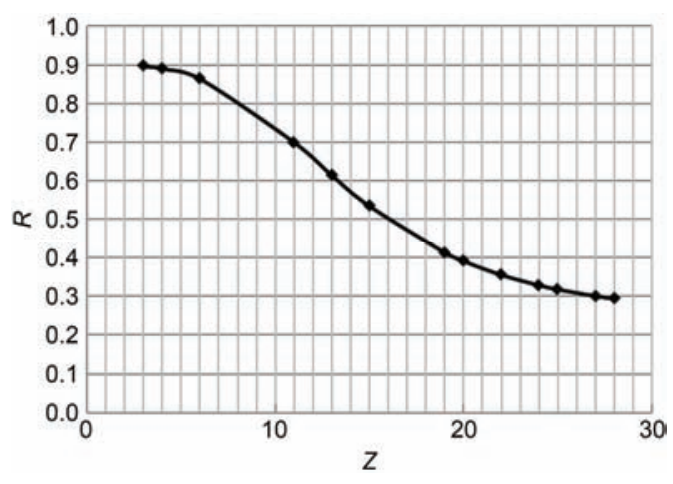

图 4100 和 $60 \mathrm{keV}$ 能量下 $R$ 值随原子序数 $Z$ 变化曲线图

Figure $4 R$ decreases monotonically with the increment of atomic number, the values are calculated with two X-ray energies of 60 and $100 \mathrm{keV}$

(a)

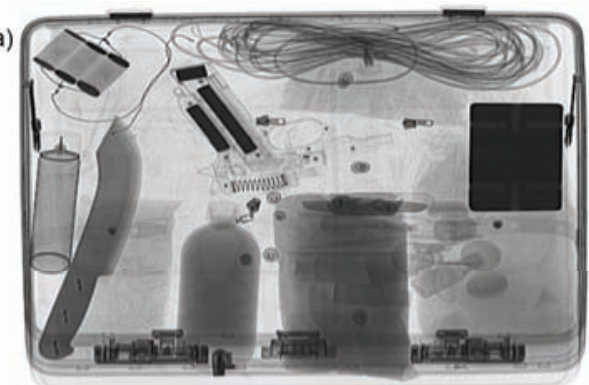

(b)

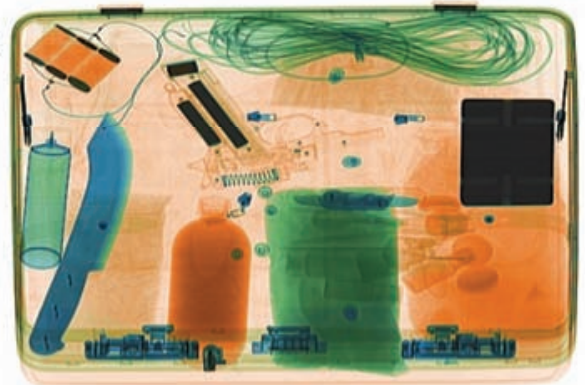

图 5 单能透视图像(a)和双能透视图像(b)对比

Figure 5 Comparison of single-energy (a) and dual-energy (b) X-ray transmission image

$\mathrm{X}$ 射线透视成像技术在货物以及旅客行李物品 安全检查中都有着广泛的应用. 一个典型的成像装 置由射线源、探测器阵列、待检物体及传送装置、计 算机控制和成像设备等组成(图6). 其中, 单能X射线 成像技术是最早应用于安全检查的技术之一, 但是 对于材料分辨能力的缺失使其难以实现固、液态炸药 等危险物质的查验. 随着透视成像技术的进步, 逐渐 发展出双能透视成像、多视角成像等新技术 ${ }^{[6]}$, 极大 地提升了材料分辨及爆炸物的检出能力, 因此得到 了迅速普及. 而针对港口、海关等的大型集装箱查验 需求, 由于穿透 $\mathrm{X}$ 射线能量需达到兆电子伏级, 不

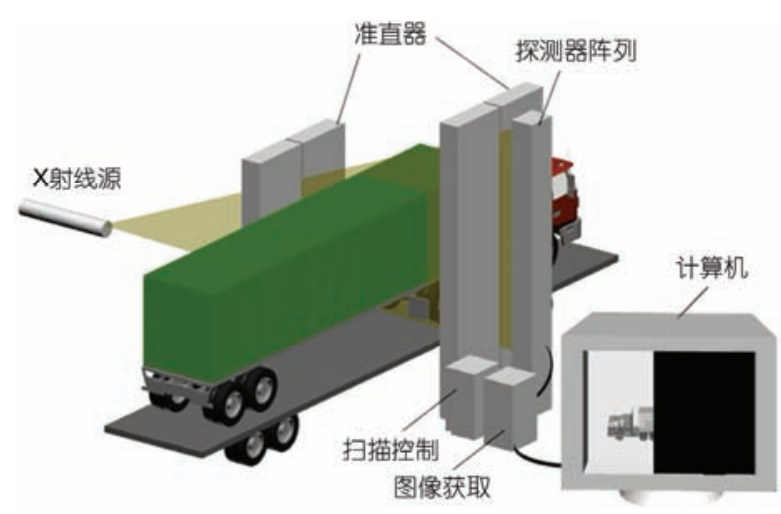

图 6 集装箱X射线透视成像系统示意图

Figure 6 Illustration of a X-ray transmission imaging inspection system for sea freight container

同材料在此能量段内对X射线的衰减差异很小, 很难 实现材料分辨 ${ }^{[7]}$. 清华大学康克军等在国际上首次提 出了基于能谱整形及图像相似性聚类的高能双能成 像及材料识别方法, 实现了无机物、有机物、轻金属 及重金属四类物质的有效区分 ${ }^{[8],}$ 为新一代大型货物 内特异物质的检测提供了新思路, 并成功研制了世 界首套具备特殊材料识别功能的集装箱/车辆检查系 统(图7) ${ }^{[9]}$.

\section{2 背散射成像技术}

$\mathrm{X}$ 射线穿过物体时通过康普顿效应产生大量散 射光子, 其作用过程如图8所示, 图中散射光子的运 动方向和能量都发生了变化, 根据动量能量守恒原 理, 可以得到散射光子的能量 $E^{\prime}$ 和散射角 $\theta$ 的对应关 系:

$$
\begin{gathered}
E^{\prime}(\theta)=\frac{E}{1+\alpha(1-\cos \theta)}, \\
\alpha=\frac{E}{m_{0} c^{2}},
\end{gathered}
$$

其中 $E$ 为人射光子能量, $m_{0}$ 为电子静止质量, $c$ 为光速. 设 $e$ 为电子电荷量, 则散射光子的角分布可以由 Klein-Nihsina经验公式计算得到:

$$
\frac{\mathrm{d} \sigma_{c}}{\mathrm{~d} \Omega}=\frac{1}{2} r_{e}^{2} P(E, \theta)^{2}\left[P(E, \theta)+P(E, \theta)^{-1}-\sin ^{2} \theta\right],
$$

其中 $r_{e}$ 为经典电子半径, $P(E, q)$ 为散射前后光子 能量比值:

$$
P(E, \theta)=\frac{1}{1+\left(E / m_{0} c^{2}\right)(1-\cos \theta)} .
$$



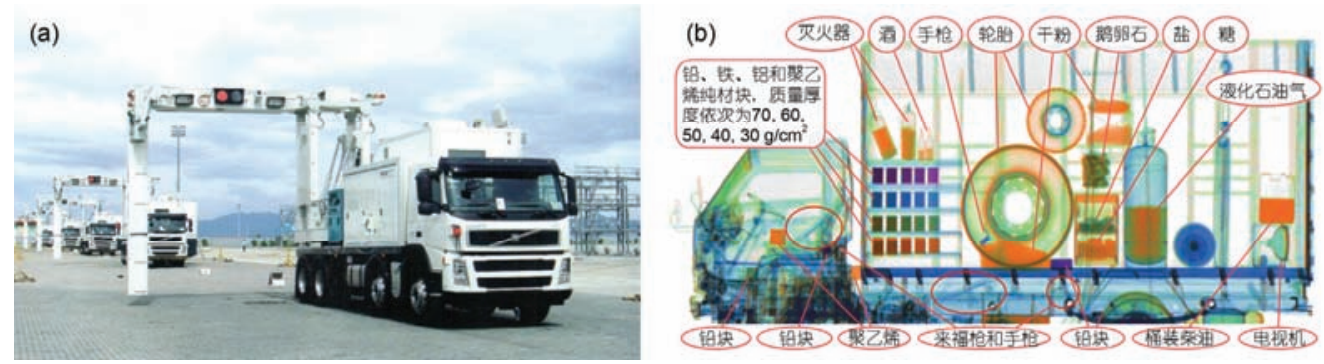

图 7 移动式集装箱/车辆检查系统(a)及其双能X射线成像图像(b)

Figure 7 Mobile container/vehicle inspection system (a) and a dual energy X-ray image generated by it (b)

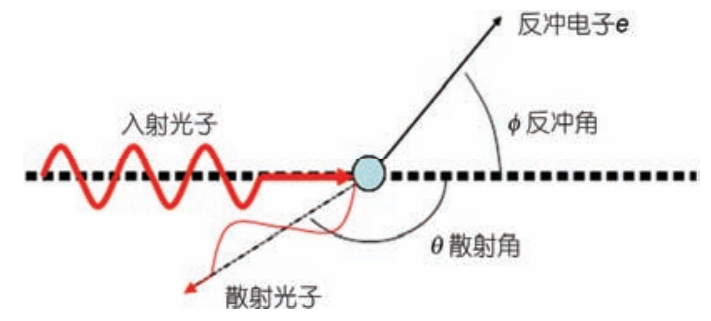

图 8 康普顿散射原理示意图

Figure 8 Diagram of Compton scattering

通过对反向散射光子进行探测成像的技术称为 背散射成像. 如图9所示, 成像时, 散射光子探测器 和射线源设置于物体的同一侧以避免透射射线的干 扰, 并配有准直器或成像孔径以获得清晰的图像. 背 散射成像的关键在于飞点扫描技术, 它通过设置在 飞轮上的笔束准直器产生笔束 X射线, 并使飞轮按照 一定的速度旋转, 使射线束沿坚直方向周期性扫描, 结合物体运动(或扫描装置运动)最终实现对目标物 体的二维逐列扫描成像 ${ }^{[10]}$. 由于散射光子在传播路 径上会进一步受物体阻挡而发生衰减, 而高原子序
数的物质造成的这种衰减会更加显著, 因此具有较 低原子序数的物质在背散射图像中具有更高的亮度, 这使得背散射对有机物和无机物的区分更加容易， 同时也更有助于爆炸物这类具有低原子序数的材料 的检出. 另一方面, 对于距离探测器较远的区域, 探 测器能接收到的散射信号更弱, 这使得背散射技术 更容易检测物体浅表层的信息，从图像上可以看出 物体位置的远近差异造成的图像亮度变化, 这是背 散射不同于透视成像的另一主要特征. 图 10所示为 背散射成像和透视成像的对比示例, 实际的成像系 统往往将背散射和透视技术相结合, 以得到更丰富 的被检测物的特征, 相应的综合成像技术目前现已 在集装箱检查、车辆检查、行李物品检查以及人体安 检等方面得到广泛应用 ${ }^{[11,12]}$.

实际应用中, 由于背散射扫描的射线源和探测 装置都在物体同一侧, 因此整套成像系统可以设计 和安装在车辆中, 成为移动式检测装置, 当背散射装 置从可疑车辆、箱体一侧通过, 即可获得实时扫描图 像. 这种移动式检测系统实现了良好的隐蔽性和机

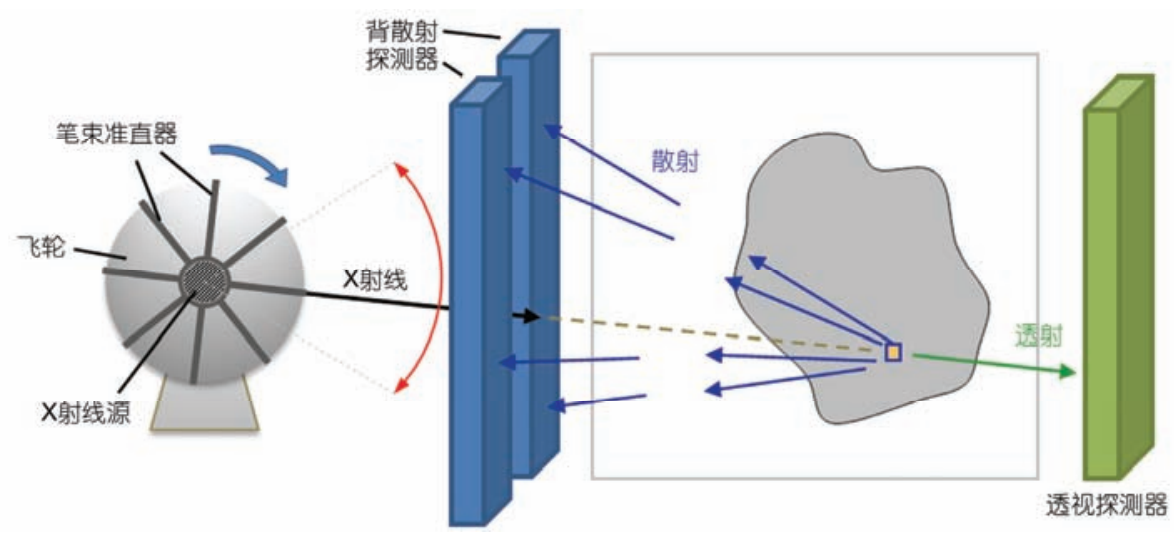

图 $9 X$ 射线背散射成像原理

Figure 9 Illustration of X-ray backscatter imaging system 

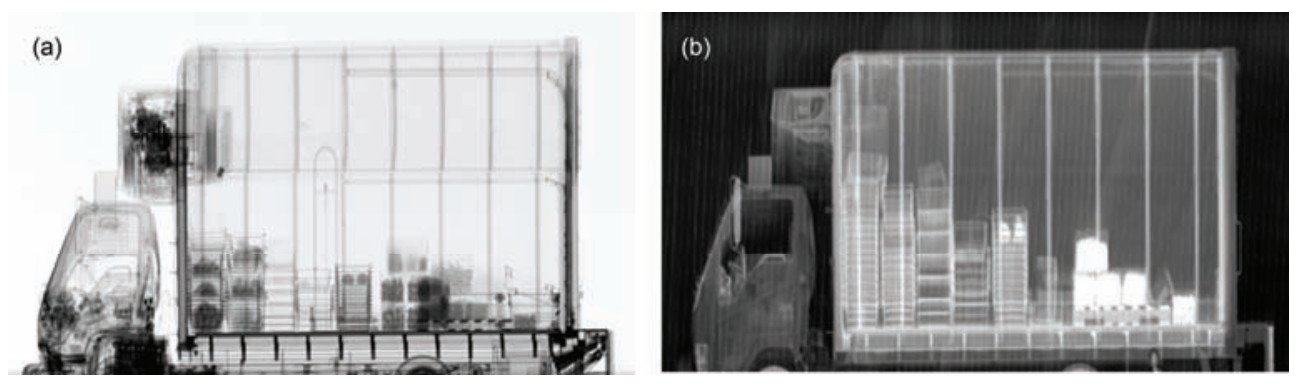

图 10 透视和背散射成像图像对比 ${ }^{[13]}$. (a) 透视图像; (b) 背散射图像

Figure 10 Comparison of X-ray transmission and backscatter image of a lorry ${ }^{[12]}$. (a) Transmission image; (b) backscatter image

动性, 能够有效应对各类场所的突发应急检查及反 恐排查. 此外, 除了针对货物、车辆, 针对人体的检 查同样是安检的重要组成. 利用高密度物质的背散 射信号弱于人体皮肤的背散射信号这一原理, 可以 实现人体背散射成像. 检查时, 使用较低能量、剂量 的X射线束扫描人体, 根据扫描产生的灰度图像信息 判断被检查者是否存在夹藏(图11). 人体背散射成像 可以实现包括塑料炸弹、刀具、枪支、毒品等违禁品 的检出, 是高效的人体检测手段.

\section{X-CT成像技术}

由于X射线透视检查设备存在物品影像重叠、图 像失真的问题, 难以清哳展现复杂行包中的物品结 构信息. 20 世纪70年代, Hounsfield等人发明了X射线 计算机断层成像 (CT)技术, 如图 12所示, 它通过对

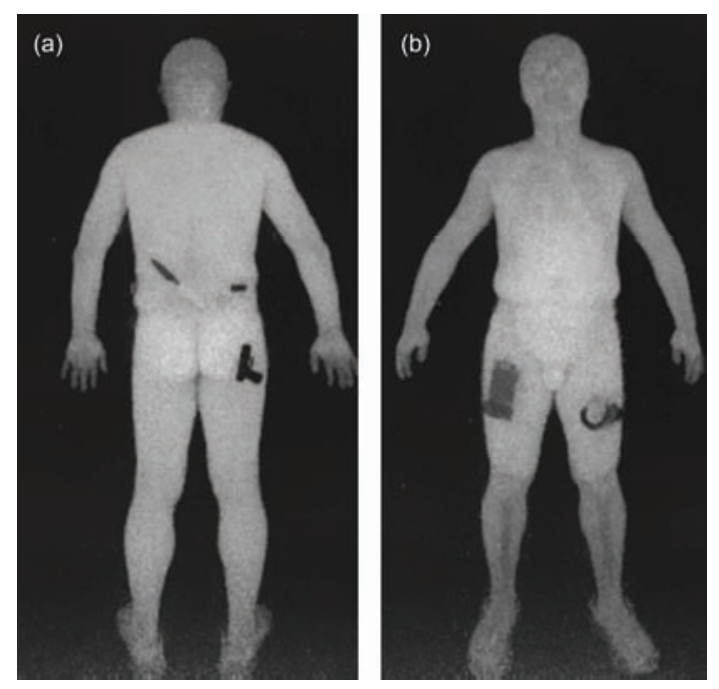

图 11 人体背散射图像, 可以发现藏于衣物内的刀具、枪支等特殊 物品

Figure 11 Hidden weapons shown in low image densities can be clearly discriminated on full body X-ray backscatter images
目标物体进行全方位的三维扫描，利用计算机重建 获得被检物体内部三维结构信息, 有效提升了违禁 品识别率和查验效率. X射线 CT技术在成像模式、扫 描速度和可靠性等方面的突出优势, 被公认是传统X 射线透视成像的替代技术，成为目前机场、海关等用 于行包检查的新手段.

CT成像的数学理论于 1917 年由奥地利数学家 Radon证明: 如果可以获得物体内某个切面沿不同方 向的密度数值的线积分, 那么通过投影变换可计算 出该切面内物体的密度分布. 这是最早的从投影重 建图像的数学理论, 奠定了 $\mathrm{CT}$ 重建理论的基石. 以 二维平行束CT扫描为例(图13), 若 $\mu(x, y)$ 为待重建物 体图像, $p(\theta, t)$ 为在 $\theta$ 投影角下的一组平行束投影(线 积分), 那么 $\mu(x, y)$ 和 $p(\theta, t)$ 存在如下关系,

$$
\int p(\theta, t) \mathrm{e}^{-\mathrm{i} 2 \pi \rho t} \mathrm{~d} t=\iint \mu(x, y) \mathrm{e}^{-\mathrm{i} 2 \pi \rho(x \cos \theta+y \sin \theta)} \mathrm{d} x \mathrm{~d} y .
$$

CT重建算法不断发展沿革至今, 主要可分为解 析重建算法和迭代重建算法. 前者主要基于傅里叶 中心切片定理(即式(8)), 包括滤波反投影算法、超短 扫描算法、PI线重建算法、Cone-parallel、FDK等三 维近似重建算法以及Katsevich的三维精确重建算法 等 ${ }^{[14-21]}$, 后者则将投影方程近似描述成线性方程组, 通过建立物理模型和引人先验信息的方法进行迭代 求解，常见的如代数迭代算法、统计迭代算法、基于 稀疏表达的最优化重建方法等 ${ }^{[2225]}$.

对于安检扫描, 由于待检物质材料种类繁多、形 状尺寸各异, CT重建图像中往往会受到难穿透物质 的影响, 形成各种伪影. 同时, 快速扫描成像的需求 也对 CT重建的运算速度提出了很高的要求. 因此近 年来针对重建方法的研究主要集中在伪影消除、缺失 数据补偿以及快速重建等方向. 其中Mouton等人 ${ }^{[26,27]}$ 就主流 CT金属伪影校正方法对安检 $\mathrm{CT}$ 图像的校正效 

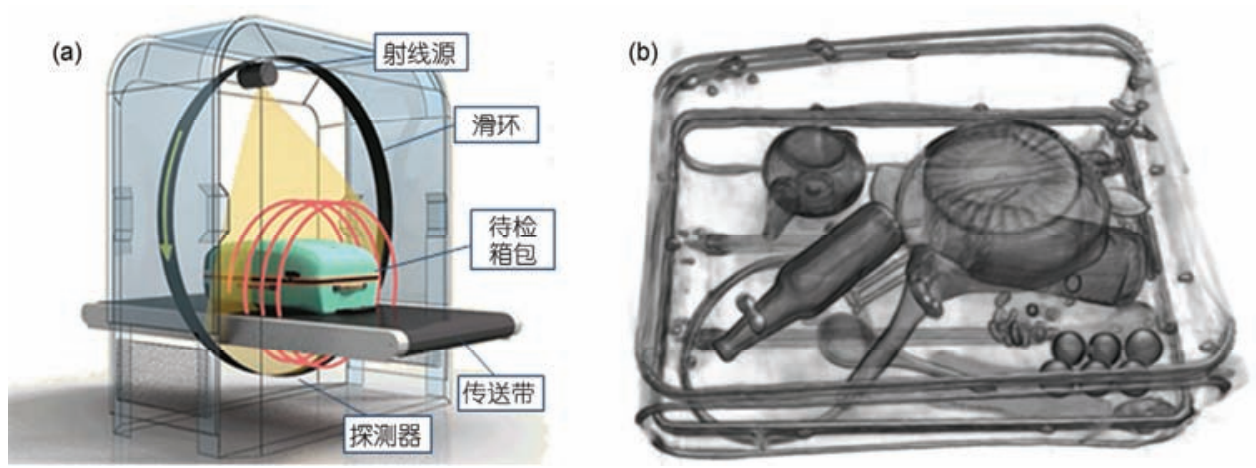

图 $12 \mathrm{X}$ 射线CT成像系统示意图(a)和CT三维重建图像(b)

Figure 12 Illustration of X-ray CT inspection system (a) and a reconstructed 3D image (b)

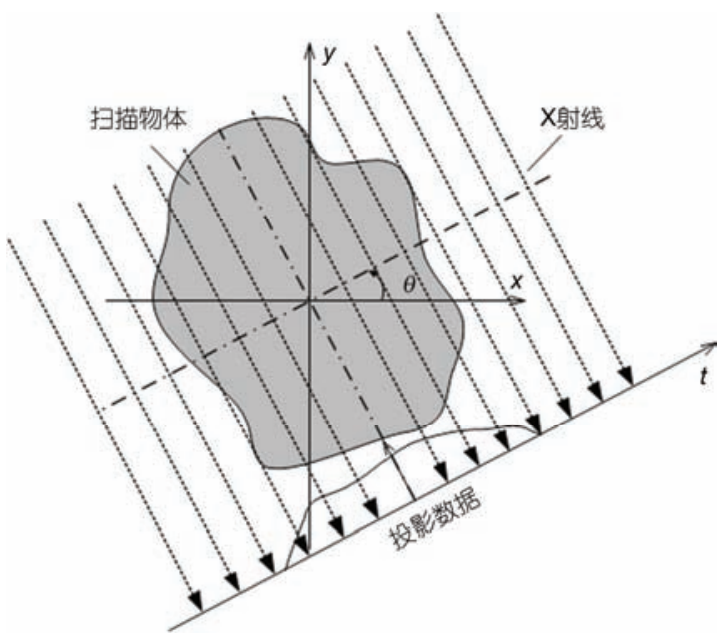

图 13 CT二维平行束投影示意图

Figure 13 Coordinate system for 2D parallel-beam imaging
果进行了评测, 并提出通过非局部去噪法以及阈值约 束法提升金属伪影校正效果, Fuchs等人 ${ }^{[28]}$ 尝试使用压 缩感知迭代重建方法实现少视角扫描下的集装箱 CT 成像, 金金金等人 ${ }^{[29]}$ 提出使用多能谱投影及参数化物体 模型的方法实现对金属容器杯状伪影的校正. 而针对 安检 $\mathrm{CT}$ 快速成像这一研究热点, 张丽等人 ${ }^{[30]}$ 首次提 出了大螺距多排螺旋 $\mathrm{CT}$ 安检成像方法和系统, 在国 内率先实现了大层厚、快速扫描技术，高河伟等人 ${ }^{[31]}$ 提出一种基于Linogram算法的 CT成像方法及系统，通 过直线轨迹的扫描模式实现快速 CT成像(图 14).

近年来静态 CT技术在高速扫描方面的革命性优 势, 逐渐成为安检 $\mathrm{CT}$ 成像研究和系统设计的焦点. 为了满足不断增长的客运、货运需求, 更高速度的安 检 CT扫描是目前亟待解决的关键问题，传统的螺旋

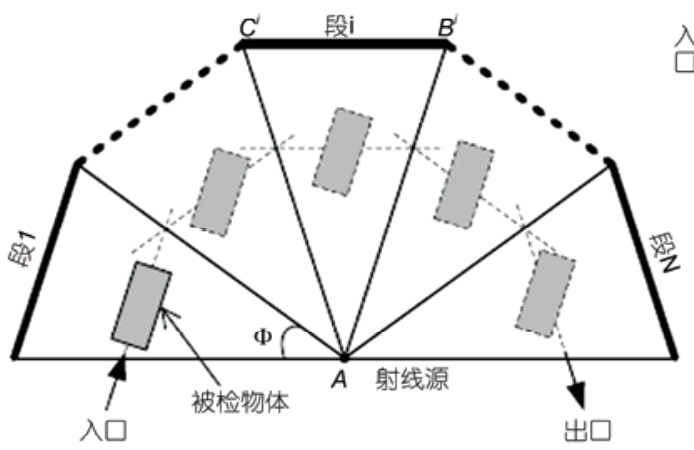

(a)

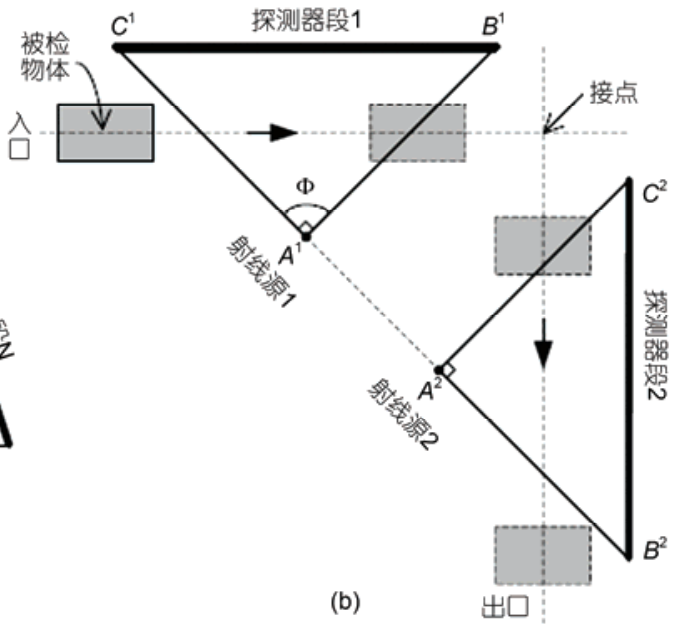

图 14 直线CT扫描系统示意图

Figure 14 Illustrations of straight-line CT scan 
CT依靠滑环快速旋转实现高速扫描, 受环上过高的 离心加速度以及高压绝缘材料的应力限制, 正逐渐接 近其转速的极限 ${ }^{[32]}$. 静态 CT利用分布在物体周围的 一系列 X射线源依次出束实现CT扫描, 系统结构如图 15 所示 ${ }^{[33,34]}$. 它通过射线源高速切换, 可实现每秒几 十转的等效转速, 是滑环 CT转速的几倍甚至几十倍, 不但减少了噪音和振动, 也避免了过高的向心加速度, 整体系统更为安全、可靠. 碳纳米管冷阴极 X射线源技 术的研究, 使得密集排布的多焦点X射线源模块成为 可能, 进一步推动了静态CT技术的发展 ${ }^{[35 ~ 37]}$.

\section{4 双能CT技术}

结合双能技术是CT应用于安检的一个突出优势. 不同于双能透视成像, 双能 CT可以对被检物的成分 进行准确的定量分析, 其技术的关键在于结合双能 分解和 CT 重建技术获得物质等效原子序数和电子密 度的空间分布. 通常, 行李物品扫描所使用的能量范 围内X射线穿透物体主要发生光电效应和康普顿效 应, 物体的线性衰减系数可以表示为上述两种效应 的叠加:

$$
\begin{gathered}
\mu=\left(\sigma_{p h}+\sigma_{c}\right) n \approx a_{1} f_{p h}+a_{2} f_{K N}, \\
a_{1}=\rho_{e} Z^{3} / 2, a_{2}=\rho_{e} / 2,
\end{gathered}
$$

其中 $\sigma_{p h}$ 和 $\sigma_{c}$ 分别为光电效应和康普顿效应截面, $n$ 为 原子密度, $f_{p h}$ 和 $f_{K N}$ 分别为 $\sigma_{p h}$ 和 $\sigma_{c}$ 同人射 $\mathrm{X}$ 射线能量相 关的经验公式, $\rho_{e}$ 为物质的电子密度. 由于 $\alpha_{1}$ 和 $\alpha_{2}$ 均 和 X射线人射能量无关, 因此通过两种不同能量的 $\mathrm{X}$ 射线的投影, 可以得到 $\alpha_{1}$ 和 $\alpha_{2}$ 的投影方程组, 最终求 解出物质的原子序数和电子密度分布 ${ }^{[38]}$. 这种将物 质对人射 X射线的吸收过程划分为两种效应的分解

方法，称为双效应分解方法. 此外，目前同样被大量 应用的另一种双能分解方法被称为基材料分解法, 它通过将衰减系数假设为某两种主要物质的衰减系 数的线性组合来实现双能分解. 基材料分解法的准 确性依赖于基材料的选择，如果被检测物体的衰减 系数在两种基材料中间, 那么基材料分解法的准确 性较高, 而如果被检测物体的衰减系数处于两种基 材料的范围之外, 那么这种分解的误差可能会被迅 速放大.

目前, 主要的双能 CT 重建方法可以归为 3 类, 分

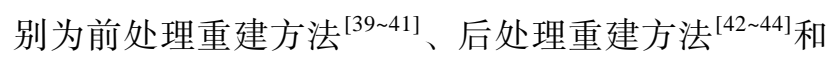
迭代重建方法 ${ }^{[45 ~ 47]}$. 其中前处理重建方法能够有效 地消除射束硬化 (即具有较宽能谱的X射线穿过物体 后, 低能光子受到更多的衰减, 射线束中高能光子占 比变多的效应)对重建结果带来的影响, 并且不受 X 射线能谱的影响, 因此得到了较为广泛的应用. 采用 双能 CT技术的爆炸物检测装置同时具备了很强的空 间分辨能力和物质分辨能力, 由于大部分的物品的 电子密度和原子序数是确定的(图16), 并且爆炸物、 毒品等违禁品明显的处于和常见物品不同的位置, 结合其空间形状信息, 双能 CT可以准确地发现并定 位行李中隐藏的违禁品, 实现自动分析及报警功能, 这是除了三维结构成像以外, CT技术替代传统透视 技术的另一个重要因素. 图17为同方威视研制的 CT 型行李安全检查设备的双能 CT 重建三维结果及切片 图像, 设备同时具有嫌疑物自动报警功能, 对可疑物 体用红框予以标记.

\section{5 能谱成像技术}

随着探测器技术的不断发展, 具有能谱分辨能

(a)

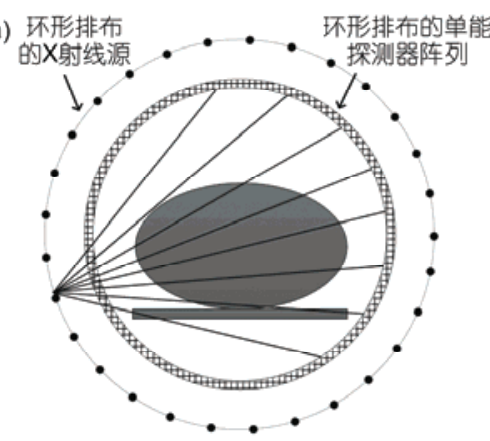

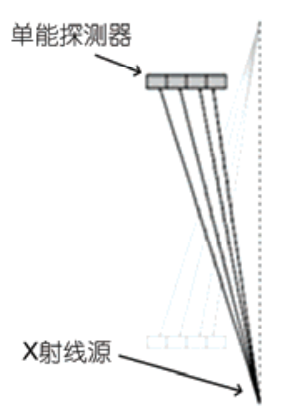

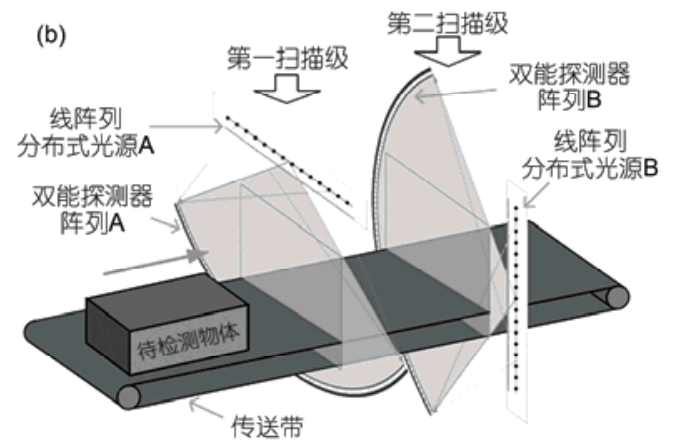

图 15 静态CT扫描系统结构示意图. (a) 单扫描面结构; (b) 多扫描面结构

Figure 15 Two mutli-source stationary CT systems. (a) X-ray sources are distributed in a circle; (b) X-ray sources are distributed in straight line segments 


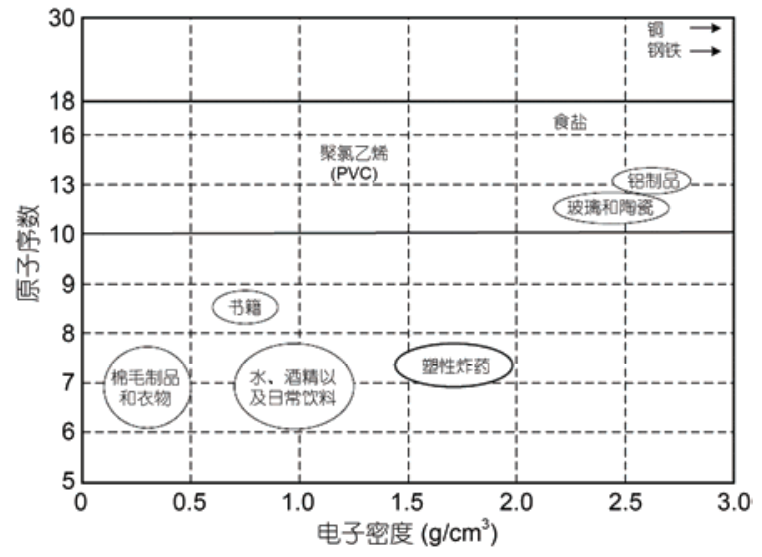

图 16 常见物质的密度和有效原子序数分布图

Figure 16 Effective atomic number and electron density data are used to identify contrabands
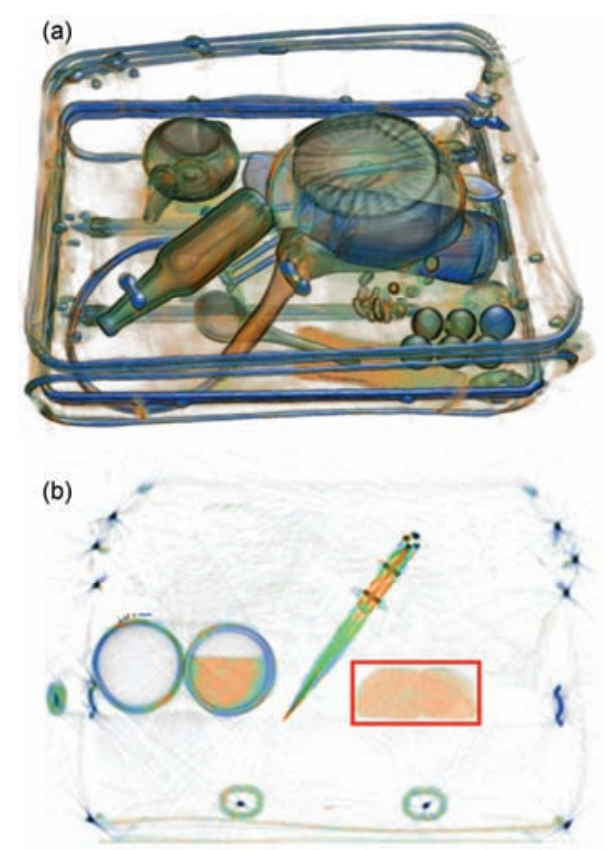

图 17 双能CT重建图像. (a) 三维重建结果; (b) 断层结果及自动标 识和报警

Figure 17 Dual-energy CT reconstruction images. (a) 3D volumerendering image; (b) 2D slice image with automatic threat detection

力的光子计数探测器成为研究的热点, 并开始在 $X$ 射 线成像领域得到应用. 光子计数探测器能够将人射 $\mathrm{X}$ 射线分为不同的能量区域进行单独计数, 一次扫描 即可获得不同能量区间的多幅衰减图像, 获得详细 的能谱信息及不同能量射线的衰减信息, 由此引出 了“能谱成像”的概念, 是未来X射线成像领域的重要 发展方向.
相对于传统的双能成像方式，能谱成像不同的 能区之间完全没有重叠, 区分度更好, 同时, 光子计 数探测器通过阈值甄别可以过滤低能噪声, 有效提 高图像信噪比, 这是传统的电荷积分式X射线探测器 所无法实现的. 近年来光子计数型探测器的研究越 来越活跃, 不同类型计数型探测器正陆续被研制出 来. 其中, 由CERN牵头联合研制的Medipix系列, 已 经在 CT 实验样机中得到了成功应用 ${ }^{[48]}$, 清华大学张 岗等人 ${ }^{[49]}$ 研制出的基于CZT探测器的多能谱计数成 像系统用于安全检查和物质识别, 已初步验证了其 可行性，此外，加拿大Redlen公司、挪威Ideas公司以 及英国Kromek等公司目前已经有部分基于CZT半导 体的光子计数探测器上市并可以根据实际应用需求 提供定制.

多能X射线成像技术应用到透视成像中，能有效 提高成像质量并降低扫描剂量. 它克服了传统双能 技术中高低能能谱区分度不大、硬化伪影严重的缺 点, 大幅度提升了成像效果和物质识别能力. Giersch 等人 ${ }^{[50]}$ 的研究表明, 结合多能权重成像算法, 多能 成像达到同样的图像质量所需辐射剂量仅为传统 CT 的 50\%. 对 CT安检系统而言, 多能谱技术的引人可 以使物质识别尤其是危险品检测精度大幅提高. 这 是因为双能分解方程组可以在多个能段下联立求解, 不但求解更加稳定, 能量区分度的提高亦使得双能 分解误差有效减少. 另外, 由于多能成像时, 各能区 之间能量没有交叉和重叠, 得到的探测数据更加接 近单能情况, 采用双能后处理重建方法也可以方便 地得到准确的等效原子序数和电子密度的空间分布. Norlin等人 ${ }^{[51]}$ 利用Medipix光子计数系统进行了物质 识别方面的研究, 对硅和铝做成的模型进行了很好 的材料分辨. 日本的Nakashima等人 ${ }^{[52]}$ 借鉴了单色光 双能重建方法, 使用 CdTe光子计数探测器, 对碳、 铝、铁和钛组成的模体进行了CT扫描并重建出原子 序数值, 实现了对物质种类的高质量识别和分辨.

\section{$6 \mathrm{X}$ 射线衍射探测技术}

1912年，劳埃等学者用实验证实了X射线与晶体 相遇时能发生衍射现象，这一现象很快被用于晶体 结构的研究, X射线衍射成像技术得到了飞速的发展. 与传统的X射线透射成像不同，X射线衍射探测技术 (XRD)测量的是物质的相干散射信息, 不同物质的分 子结构具有不同的 X射线衍射谱，该技术可以提供 


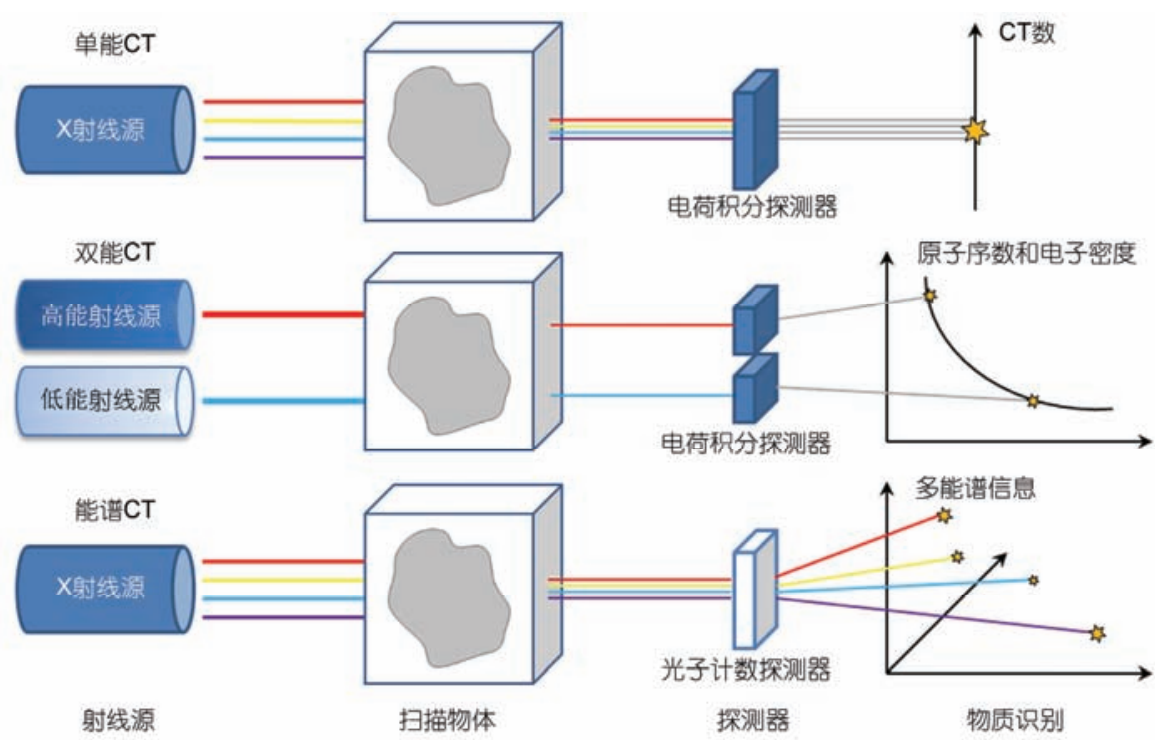

图 18 X射线能谱成像技术和传统单、双能成像技术原理对比

Figure 18 Comparison of X-ray single-energy, dual-energy and multi-energy imaging techniques

不同于X射线透射成像的物质结构信息, 为毒品、炸 药、液体危险品等违禁品的检测提供了一种全新的技 术手段, 可进一步提高现有X射线安检系统的识别准 确率 ${ }^{[53 \sim 5]}$.

根据布拉格衍射公式，相邻晶面的散射X射线发 生干涉加强的条件是

$$
2 d \sin \theta=k \lambda, k=1,2,3, \cdots
$$

其中 $d$ 为晶面间距, $\theta$ 为散射 $\mathrm{X}$ 射线和晶面夹角, $\lambda$ 为 $\mathrm{X}$ 射线波长, 如图19所示. 这一过程表明衍射过程主要 由材料晶面间距、人射射线波长和人射角决定, 因此 通过对不同 $\lambda$ 或者 $\theta$ 的散射射线强度的定量测量, 可 以得到不同晶体结构的特征信息, 进而实现对不同 晶格结构的物质的区分. 目前, X射线衍射测测量方 法大致分为两种: 即角度色散方式和能量色散方 式 $^{[56]}$. 其中角度色散方式主要测量散射 $X$ 射线强度 随 $\theta$ 的变化曲线, 实验条件要求 $\mathrm{X}$ 射线具有较强的单 色性, 测量时射线源或者探测器通过相对运动得到 不同角度下的射线强度. 由于单色光能量通常较低, 被检物体积受限, 扫描时间长, 因此该原理更多用于 实验室研究. 能量色散方式主要测量散射X射线强度 随 $\lambda$ 的变化曲线, 在系统静止的状态下即可完成物体 衍射信息的测量, 如图20所示. 该方式使用常规X射 线源, 利用能谱探测器(如CZT探测器) 测量某一固定 $\theta$ 角下不同能量的散射射线强度, 以获得待检测物体 的特征图谱(图21). 基于能量色散的XRD系统具有体

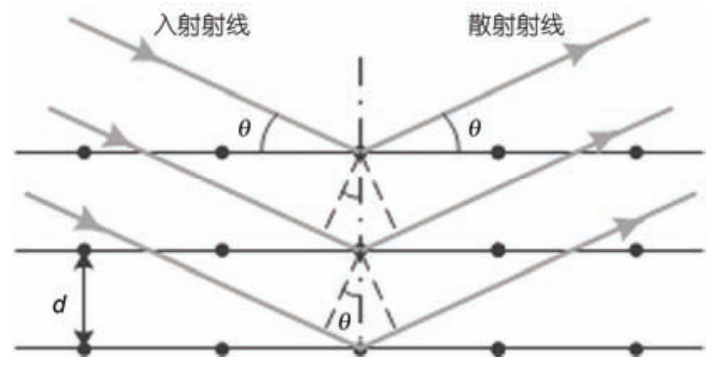

图 $19 X$ 射线穿透晶体时发生相干散射示意图

Figure 19 Reflection of X-rays by planes of atoms in a crystal
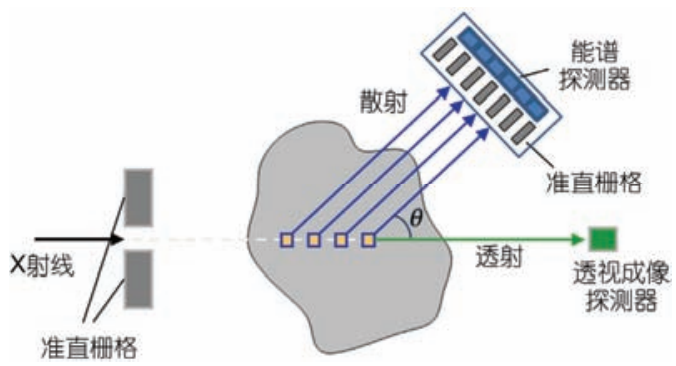

图 20 能量色散XRD系统示意图

Figure 20 A schematic diagram of the X-ray diffraction detection system

积小、系统稳定、射线穿透能力强、测量效率高等优 点, 更适用于安检等应用领域.

近10年来，国内外针对安检XRD技术展开了广 泛的研究和研发工作. 在光路设计方面, Dicken等 人 $^{[57]}$ 提出了Debye环的汇聚式设计, Harding等人 ${ }^{[58]}$ 


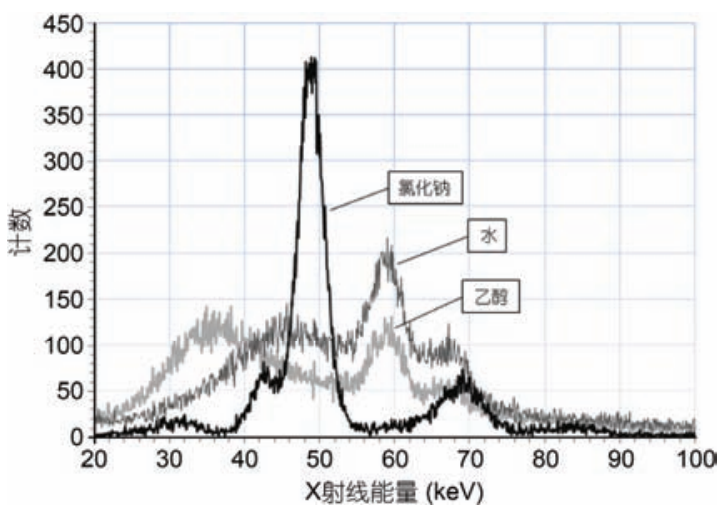

图 21 能量色散XRD系统测量的乙醇、水及氯化钠粉末的谱线

Figure 21 X-ray diffraction charts of sodium chloride powder, water and ethyl alcohol samples

提出了基于多光源的倒扇束设计, 这些成果都在不 同程度上提高了测量效率和XRD谱的信噪比. 在 XRD数据校正方面, Bomsdorf等人 ${ }^{[59]}$ 利用透射数据 对XRD谱进行校正, 降低了散射路径上物质衰减引 起XRD谱变形的影响. 在谱识别方面, 研究主要关 注PCA, LDA和 HAD等信息提取和分类方法, 实现对 违禁品的检出 ${ }^{[60,61]}$, YangDai等人 ${ }^{[62,63]}$ 通过将XRD谱 和双能 CT数据相结合, 从低信噪比的XRD谱数据中 获取液体分子特征信息, 取得了较好的实验结果.

目前, Halo, Smith Detection, Morpho等国外安检 厂商已经推出了基于能量色散的XRD安检产品, 这 类新型安检设备能够提供传送带连续扫包检查功能, 在降低误报率同时也保证了高效的检测率. 可以预 见, 随着能谱探测器等核心部件技术的日益成熟, $\mathrm{XRD}$ 技术作为一种全新的安全检测技术, 有望进一 步降低违禁品的误报率, 提高安检效率, 成为未来安 检系统中的重要组成部分.

\section{7 相衬成像技术}

传统的 $\mathrm{X}$ 射线透视成像通过物质对 $\mathrm{X}$ 射线衰减能 力的差异来获得结构信息, 但对于常见低原子序数 的物质(如棉毛制品、药物、饮料等), 由于对 $\mathrm{X}$ 射线 的衰减较弱, 使用透视成像往往得不到令人满意的 对比度. 近十多年来, X射线相衬成像方法获得了广 泛关注, 它利用X射线穿透物体时产生的相位变化进 行成像.

定义 $\delta(\vec{r})$ 为位于坐标 $\vec{r}$ 处的物质对于人射 $\mathrm{X}$ 射 线的相位因子, X射线穿过物体后发生的总的相位偏 移 $\Phi$ 可用下式表示:

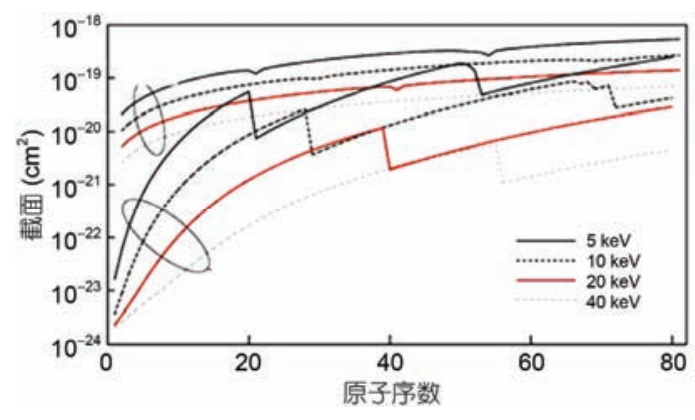

图 22 衰减截面和相位偏移截面与原子序数的关系曲线图 ${ }^{[64]}$

Figure 22 Interaction cross sections of X-ray absorption and phase shift calculated for various elements ${ }^{[64]}$

$$
\Phi_{L}=\frac{2 \pi}{\lambda} \int_{L} \delta(\vec{r}) \mathrm{d} \vec{r},
$$

其中 $\lambda$ 为 $X$ 射线波长, $L$ 为 $X$ 射线路径. 定义相位偏移 截面为 $\sigma_{p c}(\vec{r})$, 则有

$$
\sigma_{p c}(\vec{r})=\frac{2 \pi}{\lambda n} \delta(\vec{r}),
$$

其中 $n$ 为原子密度. 图 22 给出了相位偏移截面 $\sigma_{p c}$ 和衰 减截面随原子序数的变化曲线, 可以看出, 对于低原 子序数的物质, 其对 $\mathrm{X}$ 射线造成的相位偏移是衰减的 $10^{3}$ 倍, 因此通过相衬成像可以实现比衰减成像更高 的图像对比度. 此外, 由于相位偏移量 $\Phi$ 是以线积分 的形式得到的, 这意味着相衬成像也可以和衰减成 像一样通过CT扫描的方式重建相位因子的空间分布, 这极大地拓展了相衬成像的实际应用价值. 在安全 检查中，常见易燃易爆违禁品大多由低原子序数的 物质构成, 因此使用相衬成像技术理论上能够获得 更好的图像质量和信噪比, 对于提高危险品检出、保 障公共安全具有重要作用和积极意义, 因此具有广 阔的应用前景.

相衬成像技术的关键在于 $X$ 射线相位信息的提 取, 基于探测原理的差异, 目前主要发展出了晶体干 涉法、衍射增强法、同轴成像法和光栅成像法等多种 方法 ${ }^{[65 ~ 75]}$. 除了光栅成像法以外, 其余方法均对 $X$ 射 线源的单色性或者焦点大小有较高要求, 通常需借 助同步辐射等特殊X射线源装置进行成像. 2006年, Pfeiffer等人 ${ }^{[76]}$ 在当时的光栅成像法的基础上，引人 了源光栅, 并提出了基于 Talbot-Lau效应的光栅成像 系统和方法(图23), 首次实现了利用常规 X射线源进 行相袝成像; 之后, 清华大学Huang等人 ${ }^{[77]}$ 提出了基 于几何投影的非相干光栅成像技术，突破了 $\mathrm{X}$ 射线相 


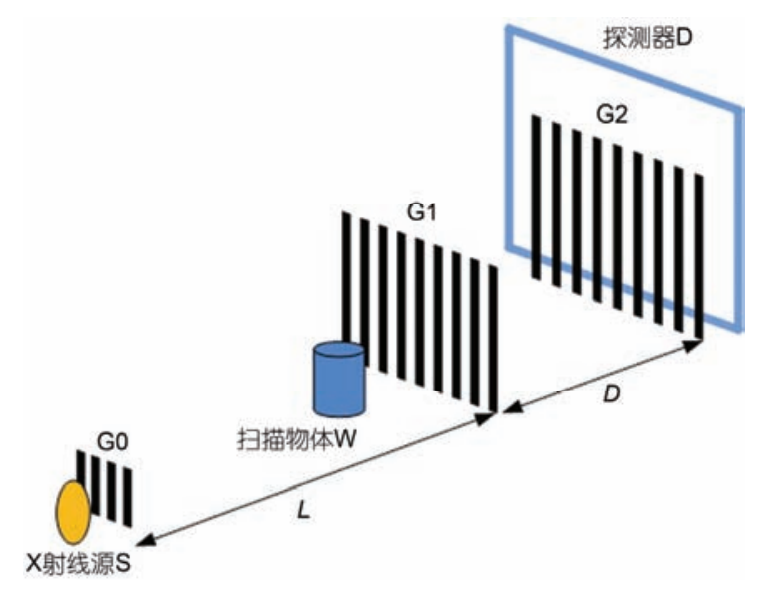

图 23 Talbot-Lau效应光栅成像系统示意图

Figure 23 Schematic setup of X-ray phase contrast imaging with TalbotLau interferometer

衬成像需要相干 $X$ 射线源的限制; Olivo等人 ${ }^{[78]}$ 提出 了基于边缘照明的非相干光栅成像技术, 这些研究 均有效降低了光栅成像技术对 $\mathrm{X}$ 射线源的要求, 大大 降低了相衬成像的实现难度, 使光栅相衬成像成为 目前应用最为广泛的相衬成像方法. 近年来, 相衬成 像在系统设计和方法研究等方面出现了大量新进展, 其中 Momose和Zanette等人 ${ }^{[79 ~ 81]}$ 分别提出二维光栅 系统以及成像方法, Thuering等人 ${ }^{[82]}$ 进行了双能光栅 成像的研究, Pelzer等人 ${ }^{[83]}$ 分别进行了基于光子计数 探测器的多能谱光栅成像方法的研究, 牛惎笨和姜 晓磊等人 ${ }^{[84,85]}$ 分别提出了使用多光源系统替代 Talbot-Lau系统中的大焦点X光源和源光栅的成像方 法, 黄志峰等人 ${ }^{[86]}$ 提出了一种基于源光栅步进的相 位步进方法, 减少了相位步进过程对高精度机械平 移装置的依赖, Modregger等人 ${ }^{[87]}$ 提出一种反卷积的 信息提取方法, 王振天等人 ${ }^{[88]}$ 提出了一种任意三点 取值的三点信息提取方法, 朱佩平等人 ${ }^{[89,90]}$ 提出了 一种正反投影信息提取方法, 这些均使得相衬成像 技术日趋成熟和完善.

目前, X射线相衬成像相关的研究工作主要集中 在生物医学成像方面, 运用于安全检查的相关研究 尚处于起步阶段. 相衬技术运用于安检的主要问题 在于其相对较慢的成像速度和相对较低的 X射线电 压, 尚难以满足安检对于扫描速度和箱包穿透力的 要求. 尽管如此, 相关研究依然得到了初步成果:
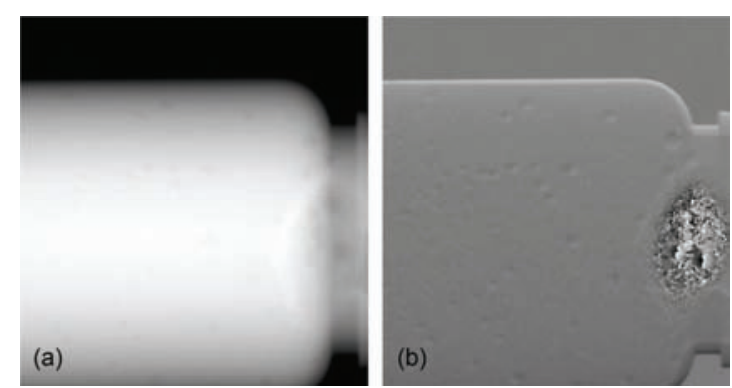

图 24 掺有粉末的沐浴露的X射线成像. (a) 透视图像; (b) 相衬图像

Figure 24 X-ray transmission image (a) and phase contrast image (b) of a lotion mixed with powder

Olivo等人 ${ }^{[91]}$ 通过实验分析了相衬成像技术应用于安 检的优势，指出相衬图像的对比度能够达到透视图 像的 7.5 倍至 40 倍; 姜晓磊等人 ${ }^{[85]}$ 提出一种基于 $X$ 射 线光栅成像技术的液体安全检查方法, 通过相衬成 像识别藏匿于均匀液体中的粉末等物质(图 24), 同 时, 提出一种两套光栅的相衬 CT物质成分识别方法, 通过结合衰减 $\mathrm{CT}$ 和相衬 $\mathrm{CT}$ 重建结果, 获得电子密度 和原子序数分布图像.

\section{8 总结及展望}

$\mathrm{X}$ 射线具有多种与物质相互作用的机制，近几十 年来根据不同的相互作用原理研究和发展出多种查 验技术, 为公共安全提供了重要和全面的保障. 本文 对 X射线技术及其安检应用研究作了全面的介绍, 针 对物理机制、关键技术和应用特点等进行了分析, 并 对新技术的研究及应用作了展望. 随着 $\mathrm{X}$ 射线源、探 测器技术、信息获取技术、成像方法等新的突破, $\mathrm{X}$ 射线检测技术得到了进一步的深化：探测精度的提 高, 检测范围的拓展, 以及查验速度的改善, 都不断 丰富着X射线安检技术的内涵, 也使得相关研究和应 用朝着更安全、更快速、更智能的方向推进. 例如微 剂量透视/散射成像技术可以更好地降低受检人员的 辐射水平; 静态 CT扫描可以替代传统滑环 CT, 提高 民航托运行李的查验速度; 能谱CT技术的发展有望 最终替代目前的双能透视成像设备, 实现安全快捷 的智能查验. 此外, 将X射线透视成像与相衬成像、衍 射XRD成像等检查技术进行有效融合也是未来 X射 线安检应用的热点方向. 这些研究都将进一步推动 $\mathrm{X}$ 射线技术在公共安全领域发挥更加重要的作用. 


\section{参考文献}

1 National consortium for the study of terrorism and responses to terrorism, Annex of Statistical Information Country Reports on Terrorism 2014, http://www.state.gov/documents/organization/225043.pdf

2 Global Terrorism Index 2015, http://economicsandpeace.org/wp-content/uploads/2015/11/Global-Terrorism-Index-2015.pdf

3 Xu W C. Encyclopedia Britannica International (Chinese Edition) (in Chinese). Beijing: Encyclopedia of China Publishing House, 1999. [徐唯成. 不列颠百科全书国际中文版. 北京: 中国大百科全书出版社, 1999]

4 Thomas A, Banerjee A. The History of Radiology. Oxford: Oxford University Press, 2013

5 Edison T A. Early days of X-ray revisited. http://www.auntminnieeurope.com

6 Zheng J Z, Lu S D. The application of X-ray technology in sectuirty inspection domain (in Chinese). CT Theory Appl, 2012, 21: 357-364 [郑金州, 鲁绍栋. X 射线技术在安检领域的应用. CT 理论与应用研究, 2012, 21: 357-364]

7 Moore J F, Ravichandran V C, Reiter R F. Better imaging: the key to better cargo inspection. Port Technology International 10th Edition, 2006, 4: 113

8 Chen Z, Wang X W. Cargo X-ray imaging technology for material discrimination. Port Technology International 13th Edition, 2009, 30: 163

9 Sessler A, Wilson E. Engines of Discovery: A Century of Particle Accelerators. Singapore: World Scientific, 2014

10 Herr M D, Mclnerney J, Lamser D G, et al. A flying spot X-ray system for Compton backscatter imaging. IEEE Trans Med Imag, 1994, 13: 461-469

11 Liu S, Jin H. X-ray security inspection techniques (in Chinese). J Chin People's Public Secur Univ (Sci Tech Ed), 2008, 58: 78-80 [刘舒, 金华. X 射线安全检查技术. 中国人民公安大学学报(自然科学版), 2008, 58: 78-80]

12 Wang Y. X-ray backscatter imaging technique in security applications (in Chinese). China Secur Protect, 2012, 1-2: 118-121 [王勇. X 射 线背散射成像技术在安检中的应用. 中国安防, 2012, 1-2: 118-121]

13 American Science \& Engineering, http://www.as-e.com

14 Gao S K. Medical imaging system (in Chinese). Beijing: Tsinghua University Press, 2000 [高上凯. 医学成像系统. 北京: 清华大学出 版社, 2000]

15 Kudo H, Noo F, Defrise M, et al. New super-short-scan algorithms for fan-beam and cone-beam reconstruction. IEEE NSS/MIC Conference, 2002

16 Zou Y, Pan X. Exact image reconstruction on PI-lines from minimum data in helical cone-beam CT. Phys Med Biol, 2004, 49: 941-959

17 Tang X, Hsieh J, Nilsen R A, et al. A three-dimensional-weighted cone beam filtered backprojection (CB-FBP) algorithm for image reconstruction in volumetric CT-helical scanning. Phys Med Biol, 2006, 51: 855-874

18 Feldkamp L A, Davis L C, Kress J W. Practical cone-beam algorithm. J Opt Soc Am, 1984, A1: 612-619

19 Wang G, Lin T H, Cheng P C, et al. A general cone-beam reconstruction algorithm. IEEE Trans Med Imag, 1993, 12: 486-496

20 Katsevich A. Analysis of an exact inversion algorithm for spiral cone-beam CT. Phys Med Biol, 2002, 47: 2583-2598

21 Katsevich A. Theoretically exact filtered backprojection-type inversion algorithm for spiral CT. SIAM J Appl Math, 2002, 62: 2012-2026

22 Zeng G L. Medical Image Reconstruction a Conceptual Tutorial. Beijing: Higher Education Press, 2010

23 Xu Q, Mou X Q, Wang G. Statistical interior tomography. IEEE Trans Med Imag, 2011, 30: 1116-1128

24 Xu Q, Yu H, Mou X, et al. Low-dose X-ray CT reconstruction via dictionary learning. IEEE Trans Med Imag, 2012, 31: 1682-1697

25 Gao H, Yu H, Osher S, et al. Multi-energy CT based on a prior rank, intensity and sparsity model (PRISM). Inverse Probl, 2011, 27: 115012

26 Mouton A, Megherbi N, Slambrouck K V, et al. An experimental survey of metal artefact reduction in computed tomography. J X-ray Sci Technol, 2013, 21: 193-226

27 Mouton A, Megherbi N, Flitton G T, et al. A novel intensity limiting approach to metal artifact reduction in 3D CT baggage imagery. In: 19th IEEE International Conference on Image Processing (ICIP), 2012

28 Fuchs T, Schön T, Dittmann J, et al. Recent progress in 3-D imaging of sea freight containers. The 41st Annual Review of Progress in Quantitative Nondestructive Evaluation, 2015, 34: 556-561

29 Jin X, Li L, Shen L, et al. Metal artifact correction for liquid CT scan with a parameterized image and a spectrum projection model. In: The 13th International Meeting on Fully Three-Dimensional Image Reconstruction in Radiology and Nuclear Medicine, 2015

30 Zhang L, Chen Z Q, Hu H F, et al. Test system with a CT scanner and an additional scan imaging device. German patent, DE 102008043210 B4, 2015 
31 Gao H, Zhang L, Chen Z, et al. Straight-line-trajectory-based X-ray tomographic imaging for security inspections: System design, image reconstruction and preliminary results. IEEE Trans Nucl Sci, 2013, 60: 3955-3968

32 Martin P, Herbert B, Bernhard K, et al. Technical principles of dual source CT. Eur J Radiol, 2008, 68: 362-368

33 Morton E, Mann K, Berman A, et al. Ultrafast 3D reconstruction for X-ray Real-Time Tomography (RTT). In: IEEE Nuclear Science Symposium Conference Record, 2009, 4077-4080

34 Zhang L, Chen Z Q, Huang Q P, et al. Computed tomography (CT) system and method (in Chinese). Chinese Patent, ZL201310739803.4, 2013 [张丽, 陈志强, 黄清萍, 等. CT 系统及方法. 中国发明专利, 201310739803.4, 2013]

35 Cao G, Lee Y Z, Peng R, et al. A dynamic micro-CT scanner based on a carbon nanotube field emission X-ray source. Phys Med Biol, 2009, 54: 2323-2340

36 Zhang J, Yang G, Lee Y Z, et al. Multiplexing radiography using a carbon nanotube based X-ray source. Appl Phys Lett, 2006, 89: 064106

37 Chen Y, Chen T, Gui J B, et al. Development of CNT cathode based X-ray source and imaging technology (in Chinese). CT Theory Appl, 2013, 22: 363-372 [陈圭, 陈婷，桂建保，等. 基于碳纳米管阴极的 X 射线源与成像技术进展. CT 理论与应用研究, 2013, 22: 363-372]

38 Zhang L, Chen Z, Zhang L, et al. Exact reconstruction for dual energy computed tomography using an HL curve method. In: IEEE Nuclear Science Symposium Conference Record, 2006, 6: 3485-3488

39 Wong C, Huang H. Calibration procedure in dual-energy scanning using the basis function technique. Med Phys, 1983, 10: 628-635

40 Alvarez R, Seppi E. A comparison of noise and dose in conventional and energy selective computed tomography. IEEE Trans Nucl Sci, 1979, 26: 2853-2856

41 Zhang G W, Zhang L, Chen Z Q. An H-L curve method for material discrimination of dual energy X-ray inspection systems. In: Proceedings of the IEEE Nuclear Science Symposium, 2005. 326-328

42 Steenbeek J C, Kuijk C, Grashuis J L, et al. Selection of fat-equivalent materials in postprocessing dual-energy quantitative CT. Med Phys, 1992, 19: 1051-1056

43 Heismann B J, Leppert J, Stierstorfer K. Density and atomic number measurements with spectral X-ray attenuation method. J Appl Phys, 2003, 94: 2073-2079

44 Christ G. Exact treatment of the dual-energy method in CT using polyenergetic X-ray spectra. Phys Med Biol, 1984, 29: 1501-1510

45 Yan C H, Whalen R T, Beaupré G S, et al. Reconstruction algorithm for polychromatic CT imaging: Application to beam hardening correction. IEEE Trans Med Imag, 2000, 19: 1-11

46 Fessler J A, Elbakri I, Sukovic P, et al. Maximum-likelihood dual-energy tomographic image reconstruction. Proc SPIE, 2002, 4684: 38-49

47 Sukovic P, Clinthorne N. Penalized weighted least-squares image reconstruction for dual energy X-ray transmission tomography. IEEE Trans Med Imag, 2000, 19: 1075-1081

48 Davidson D W, Watt J, Tlustos L, et al. Detective quantum efficiency of the Medipix pixel detector. IEEE Trans Nucl Sci, 2003, 50: 1659-1663

49 Zhang L, Li Y J, Zheng X C, et al. Multi-energy detection using CdZnTe semiconductor detectors. In: Proceedings IEEE Nuclear Science Symposium, 2008. R13-5: 428-433

50 Giersch J, Niederlöhner D, Anton G. The influence of energy weighting on X-ray imaging quality. Nucl Instrum Methods Phys Res Sect A: Accel Spectrom Detect Assoc Equip, 2004, 531: 68-74

51 Norlin B, Manuilskiy A, Nilsson H E, et al. Material recognition with the Medipix photon counting colour X-ray system. Nucl Instrum Methods Phys Res Sect A: Accel Spectrom Detect Assoc Equip, 2004, 531: 265-269

52 Nakashima T, Morii H, Neo Y, et al. Application of CdTe photon-counting X-ray imager to material discriminated X-ray CT. Proc SPIE, 2007, 6706: 67060C

53 Singh S, Singh M. Explosives detection systems (EDS) for aviation security. Signal Proc, 2003, 83: 31-55

54 Crespy C, Duvauchelle P, Kaftandjian V, et al. Energy dispersive X-ray diffraction to identify explosive substances: Spectra analysis procedure optimization. Nucl Instr Methods Phys Res Sect A: Accel Spectrom Detect Assoc Equip, 2010, 623: 1050-1060

55 Peterzol A, Duvauchelle P, Kaftandjian V, et al. Modeling-based optimization study for an EDXRD system in a portable configuration. Nucl Instr Methods Phys Res Sect A: Accel Spectrom Detect Assoc Equip, 2011, 654: 450-463

56 Ghammraoui B, Tabary J, Pouget S, et al. New software to model energy dispersive X-ray diffraction in polycrystalline materials. Nucl Instr Methods Phys Res Sect A: Accel Spectrom Detect Assoc Equip, 2012, 664: 324-331

57 Dicken A J, Evans J P O, Rogers K D, et al. Energy-dispersive X-ray diffraction using an annular beam. Opt Express, 2015, 23: 13443-13454

58 Harding G, Fleckenstein H, Kosciesza D, et al. X-ray diffraction imaging with the Multiple Inverse Fan Beam topology: Principles, performance and potential for security screening. Appl Rad Isotopes, 2012, 70: 1228-1237

59 Bomsdorf H, Müller T, Strecker H. Quantitative simulation of coherent X-ray scatter measurements on bulk objects. J X-Ray Sci Technol, 2004, 12: 83-96

$60 \mathrm{Yu}$ J, Tian Q, Rui T, et al. Integrating discriminant and descriptive information for dimension reduction and classification. IEEE Trans Circuits Syst Video Technol, 2007, 17: 372-377

61 Zhong Y, Zhang F, Li W, et al. A new application of X-ray scattering using principal component analysis classification and identification 
of liquid precursor chemicals. X-Ray Spectr, 2013, 42: 45-51

YangDai T, Zhang L. Liquid contrabands classification based on energy dispersive X-ray diffraction and hybrid discriminant analysis. Nucl Instr Methods Phys Res Sect A: Accel Spectrom Detect Assoc Equip, 2016, 808: 128-134

63 Zhang L, YangDai T. Determination of Liquid's Molecular Interference Function based on X-Ray Diffraction and Dual-energy CT in Security Screening. Appl Rad Isot, 2016, 114: 179-187

64 Momose A, Takeda T, Yoneyama A, et al. Phase contrast X-ray imaging using an X-ray interferometer for biological imaging. Analyt Sci, 2001, 17: 527-530

65 Bonse U, Hart M. An X-ray interferometer. Appl Phys Lett, 1965, 6: 155-157

66 Momose A. Phase-contrast radiographs of non stained rat cerebellar specimen. Med Phys, 1995, 22: 375

67 Takeda M, Ina H, Kobayashi S. Fourier-transform method of fringe-pattern analysis for computer-based topography and interferometry. J Opt Soc Am, 1982, 72: 156-160 Davis T J, Gao D, Gureyev T E, et al. Phase-contrast imaging of weakly absorbing materials using hard X-rays. Nature, 1995, 373: 595-598 Chapman L D, Thomlinson W C, Johnston R E, et al. Diffraction enhanced X-ray imaging. Phy Med Biol, 1997, 42: 2015-2025 diation. Rev Scient Instr, 1995, 66: 5486-5492 Wilkins S W, Gureyev T E, Gao D, et al. Phase-contrast imaging using polychromatic hard X-rays. Nature, 1996, 384: 335-338 Chen R C, Liu P, Xiao T Q, et al. X-ray imaging for non-destructive microstructure analysis. SSRF Adv Mater, 2014, 26: 7688-7691

David C, Nöhammer B, Solak H H, et al. Differential X-ray phase contrast imaging using a shearing interferometer. Appl Phys Lett, 2002, 81: 3287-3290

74 Momose A. Phase-sensitive imaging and phase tomography using X-ray interferometers. Opt Express, 2003, 11: 2303-2314

75 Weitkamp T, Diaz A, Nohammer B, et al. Hard X-ray phase imaging and tomography with a grating interferometer. Proc SPIE, 2004, 5535: $137-142$

76 Pfeiffer F, Weitkamp T, Bunk O, et al. Phase retrieval and differential phase-contrast imaging with low-brilliance X-ray sources. Nat Phys, 2006, 2: 258-261

77 Huang Z F, Kang K J, Zhang L, et al. Alternative method for differential phase-contrast imaging with weakly coherent hard x rays. Phys Rev A, 2009, 79: 013815

78 Olivo A, Speller R. A coded-aperture technique allowing X-ray phase contrast imaging with conventional sources. Appl Phys Lett, 2007, 91: 074106

79 Momose A, Kawamot S. X-ray Talbot interferometry with capillary plates. Jpn J Appl Phys, 2006, 45: 314-316

80 Zanette I, Weitkamp T, Donath T, et al. Two-dimensional X-ray grating interferometer. Phys Rev Lett, 2010, 105: 248102

81 Kottler C, Revol V, Kaufmann R, et al. Dual energy phase contrast X-ray imaging with Talbot-Lau interferometer. J Appl Phys, 2010, 108: 114906

82 Thuering T, Barber W C, Seo Y, et al. Energy resolved X-ray grating interferometry. Appl Phys Lett, 2013, 102: 191113

83 Pelzer G, Weber T, Anton G, et al. Grating-based X-ray phase-contrast imaging with a multi energy-channel photon-counting pixel detector. Opt Express, 2013, 21: 25677-25684

84 Niu H B, Guo J C, Wang K G, et al. New X ray tube, and fabricating method (in Chinese). Chinese Patent, ZL200610062487.1, 2012 [牛 憼笨，郭金川，王凯歌，等。一种新型 X 射线管及其制作方法. 中国发明专利, ZL200610062487, 2012]

85 Jiang X L. Research on Methods and Applications for Grating-based Imaging with Conventional X-ray Sources (in Chinese). Doctoral Dissertation. Beijing: Tsinghua University, 2015 [姜晓否. 常规 X光源光栅综合成像技术方法与应用研究. 博士学位论文. 北京：清 华大学, 2015]

86 Huang Z, Chen Z, Zhang L, et al. Large phase-stepping approach for high-resolution hard X-ray grating-based multiple-information imaging. Opt Express, 2010, 18: 10222-20229

87 Modregger P, Scattarella F, Pinzer B R, et al. Imaging the ultrasmall-angle X-ray scattering distribution with grating interferometry. Phys Rev Lett, 2012, 108: 048101

88 Wang Z, Huang Z, Chen Z, et al. Low-dose multiple-information retrieval algorithm for X-ray grating-based imaging. Nucl Instr Methods Phys Res Sect A: Accel Spectrom Detect Assoc Equip, 2011, 635: 103-107

89 Zhu P, Zhang K, Wang Z, et al. Low-dose, simple, and fast grating-based X-ray phase-contrast imaging. Proc Natl Acad Sci USA, 2010, 107: 13576-13581

90 Zhu P P, Yuan Q X, Huang W X, et al. Two-dimensional imaging system and method for medical diagnosis of human body (in Chinese) Chinese patent, ZL201310111387.3，2015 [朱佩平，袁清习，黄万霞，等. 用于人体医学检测的二维成像系统及方法. 中国发明专 利, ZL201310111387.3, 2015]

91 Olivo A, Chana D, Speller R. A preliminary investigation of the potential of phase contrast X-ray imaging in the field of homeland security. J Phys D Appl Phys, 2008, 41: 225503 


\title{
Recent progress on X-ray security inspection technologies
}

\author{
CHEN ZhiQiang ${ }^{1,2}$, ZHANG Li ${ }^{1,2} \&$ JIN Xin ${ }^{1,2}$ \\ ${ }^{1}$ Department of Engineering Physics, Tsinghua University, Beijing 100084, China; \\ ${ }^{2}$ Key Laboratory of Particle \& Radiation Imaging (Tsinghua University), Ministry of Education, Beijing 100084, China \\ * Corresponding author, E-mail: czq@mail.tsinghua.edu.cn
}

Emerging terrorist attacks seriously impacted the global security situation which brings unprecedented challenges to public transportation. Effectual anti-terrorist ways to protect personal safety and assets security become an important global demand. Consequently, to improve security inspection capabilities with state-of-art technology has long been a serious issue and hot research topic. Recent years, with the rapid development of X-ray detection technology, utilizing $\mathrm{X}$-rays to inspect hidden explosives and contrabands in cargo, luggage, vehicle and on human body become both effective and efficient. Based on different X-ray interaction behaviors with matter, a variety of inspection techniques have been researched and developed which show great potentials for security applications. This paper provides a general review on different X-ray inspection techniques such as X-ray transmission imaging, backscatter imaging, phase-contrast imaging, spectral imaging, CT reconstruction, X-ray diffraction and so on. Basic physical mechanisms, research progresses and application features are investigated. And outlooks to new technologies and applications are given.

$\mathrm{X}$-ray transmission imaging detects the attenuation of X-ray flux while penetrating objects. The transmission image has a strong discrimination for high-density materials with strong $\mathrm{X}$-ray absorption, such as guns, knives, grenades, detonators and other high atomic number items. Dual-energy X-ray imaging is an important complement that provides preliminary material classification capability. The technology has been widely used for sea freight cargo and passenger luggage inspection.

X-ray back-scatter imaging detects X-rays that reflects from the scanned objects (e.g., vehicle, container and human body). It is good for imaging low density (or low atomic number) materials. The source and detectors of the scanning device are usually designed on one side of the target object, and a rotating wheel with pencil beam collimators is the key to scan in a point-by-point method.

Computed tomography (CT) inspection system generates volumetric image of scanned item. It provides high resolution 3D inner structures nondestructively and without overlap. Due to the rapid development in CT reconstruction theory, various applications for security inspection have emerged. Hot research and development topics include material classification method, dual-energy and multi-energy CT imaging, non-spiral trajectory CT scan, artifacts correction and scan speed improvement.

X-ray diffraction (XRD) technique has been widely used to identify minerals and other crystalline materials. It also shows good ability for detecting drug, explosives and hazardous liquids in security examination. Photo-counting detectors are utilized to measure scatter flux on different photon energy levels to form diffraction spectrum. Currently research on XRD for security application is focused on improving signal-to-noise ratio, reducing scanning time, material recognition method and system design.

Phase-contrast imaging (PCI) calculates X-ray phase-shift when traversing the item. For low atomic number materials the cross section of the phase-shift can achieve up to $10^{3}$ times larger than the one of attenuation. Thus it gains much better image contrast for fruits, clothes, drugs, liquids and other low atomic number items. Grating-based imaging has been regarded as the most promising PCI method because it is a first approach that requires only conventional X-ray source rather than synchrotron or micro-focus X-ray sources. Preliminary research work can be found on the security application potentials of grating-based PCI systems and methods.

security inspection, X-ray imaging, CT reconstruction, phase-contrast imaging, X-ray diffraction

doi: 10.1360/N972016-00698 